\title{
Music of Senegal and Gambia
}

A RECENT issue in the Ethnic Folkways Library Album ${ }^{1}$ consists of records of Wolof music, with an introductory pamphlet, illustrated by photographs, by David Ames. The author points out that Wolof music clearly displays the mingling of two traditions--the West African negro style with that of Muslim North Africa. These are also reflected in many other aspects of Wolof culture. The records include traditional songs sung by professional entertainers (gewel) accompanied on the kalam, a five-stringed instrument with a resonator, the strings of horsehair being stretched over a bridge and plucked with the thumb; also dance music played on drums and recorded during the celebration of a naming ceremony, and the singing of Mohammedan hymns by the followers of a holy man, accompanied by sacred drums (tabala).

\section{La Mission démographique de la Guinée Francaise}

LA mission démographique de Guinée a été entreprise afin de connaître quel est le taux d'accroissement annuel de la population. La mission devait se préoccuper de former des agents itinérants qui iraient interroger les habitants d'un échantillon de villages, afin de rechercher les éléments statistiques nécessaires. La formation de ces agents africains a duré du is novembre r954 au is janvier 1955; un nombre d'études pilotes étaient achevées, ainsi que les sondages (entrepris au 1/s) dans les centres urbains: Conakry, Kankan, Labé, Mamou. 250.000 personnes ont été interrogées, deux cent mille kilomètres parcourus par des véhicules, près de 40.000 kilomètres à pied par des agents recenseurs. Les résultats détaillés ne sont pas encore publiés, mais on estime que la population africaine de la Guinée est en voie d'expansion rapide; la progression n'est pas uniforme sur tout le territoire, mais, d'après les premiers dépouillements entrepris, cette augmentation relative annuelle, pour l'ensemble du territoire, approche $2 \%$. Liée à une natalité qui atteint trois fois celle de la métropole, la mortalité enfantile est très forte.

\section{'Madagascar 1955'2}

Deux journées d'études malgaches avaient lieu à Paris 19-20 Février I955, à l'initiative du Secrétariat Social d'Outre-Mer. Après l'allocution d'ouverture prononcée par M. J. Razafimbahiny, M. Jean-Marie Sedes (Professeur de Sociologie aux Facultés catholiques de Lille) a donné un exposé de la situation démographique et ses incidences sociales, ce qui était suivi par un rapport de M. A. Rakotondrainibe traitant de l'état actuel de la population de Madagascar. Les questions économiques ont été discutées par MM. J. Razafimbahiny et André Fangeat. M. Hubert Deschamps (Gouverneur de la France Outre-Mer, Secrétaire Géneral du Conseil Supérieur de la Recherche Sociologique Outre-Mer) dans son discours 'Devenir Politique de la grande Île ', a parlé du statut actuel du territoire et ses modifications, du problème culturel (l'élite, l'éducation, l'équilibre d'évolution), de l'organisation intérieure de l'île, des rapports entre Malgaches et Européens et de l'évolution future des rapports entre Madagascar et la France. A l'ouverture de son discours, M. Deschamps fit remarquer qu'il ne représenta ni la recherche scientifique ni le Secrétariat de l'État, mais qu'il n'engagea que soi-même et ses propres opinions. Le discours fut suivi par un rapport de M. R. W. Rabemananjara et par un débat assez vif.

I Folkways Records and Service Corp., II7 W. 46 St., New York r9, U.S.A.
2 Rapport édité par le Secrétariat Social d'OutreMer, Paris. 200 frs. 